\title{
Extracranial glioblastoma diagnosed by examination of pleural effusion using the cell block technique: case report
}

\author{
Yusuke S. Hori, MD, ${ }^{1}$ Toru Fukuhara, MD, PhD, ${ }^{1}$ Mizuho Aoi, MD, PhD, ${ }^{1}$ Kazunori Oda, MD, ${ }^{3}$ and \\ Yoko Shinno, MD²
}

\begin{abstract}
Departments of ${ }^{1}$ Neurological Surgery and ${ }^{2}$ Pathology, National Hospital Organization Okayama Medical Center, Okayama; and ${ }^{3}$ Department of Neurological Surgery, Nippon Medical School, Tokyo, Japan
\end{abstract}

\begin{abstract}
Metastatic glioblastoma is a rare condition, and several studies have reported the involvement of multiple organs including the lymph nodes, liver, and lung. The lung and pleura are reportedly the most frequent sites of metastasis, and diagnosis using less invasive tools such as cytological analysis with fine needle aspiration biopsy is challenging. Cytological analysis of fluid specimens tends to be negative because of the small number of cells obtained, whereas the cell block technique reportedly has higher sensitivity because of a decrease in cellular dispersion. Herein, the authors describe a patient with a history of diffuse astrocytoma who developed intractable, progressive accumulation of pleural fluid. Initial cytological analysis of the pleural effusion obtained by thoracocentesis was negative, but reanalysis using the cell block technique revealed the presence of glioblastoma cells. This is the first report to suggest the effectiveness of the cell block technique in the diagnosis of extracranial glioblastoma using pleural effusion. In patients with a history of glioma, the presence of extremely intractable pleural effusion warrants cytological analysis of the fluid using this technique in order to initiate appropriate chemotherapy.
\end{abstract}

https://thejns.org/doi/abs/10.3171/2017.8.FOCUS17403

KEYWORDS cell block technique; glioblastoma; pleural metastasis

$\mathrm{E}$ XTRACRANIAL metastasis of glioblastoma is an extremely rare condition, and the estimated incidence is considered to be $<2 \% .8,12,18$ Only a few reported cases have involved multiple organs, including the skin, lymph nodes, lung, pleura, liver, spleen, and bone. .,6,15-19 $^{2}$ Nauen and $\mathrm{Li}^{11}$ described the significance of cytological analysis of pleural effusion for the diagnosis of metastatic glioblastoma, but cytological analysis of fluid specimens tends to be negative because of the small concentration of cells. Conversely, several studies have documented the usefulness of the cell bock technique when using fluid specimens such as pleural, peritoneal, and pericardial effusions. ${ }^{3,4,9,21}$ In this report, we describe a patient with extracranial glioblastoma that was diagnosed by collecting a sample of massive pleural effusion via chest drainage and examining the fluid using the cell block technique.

\section{Case Report}

History and Examination

A 75-year-old woman with a history of suspected astrocytoma presented to our emergency department with the chief complaint of an altered mental status. Eight years prior to this presentation, she had developed decreased cognitive function and had presented to our neurology department. Magnetic resonance imaging had revealed a high-intensity lesion in her left frontotemporal lobe and basal ganglia on FLAIR sequences (Fig. 1A). Encephalitis had been suspected; however, negative results had been obtained by lumbar puncture and several antibody tests, including those for antiviruses and autoimmune antibodies. Gadolinium-enhanced MRI had shown no enhanced lesion (Fig. 1B). Follow-up MRI 1 month after the first pre- 

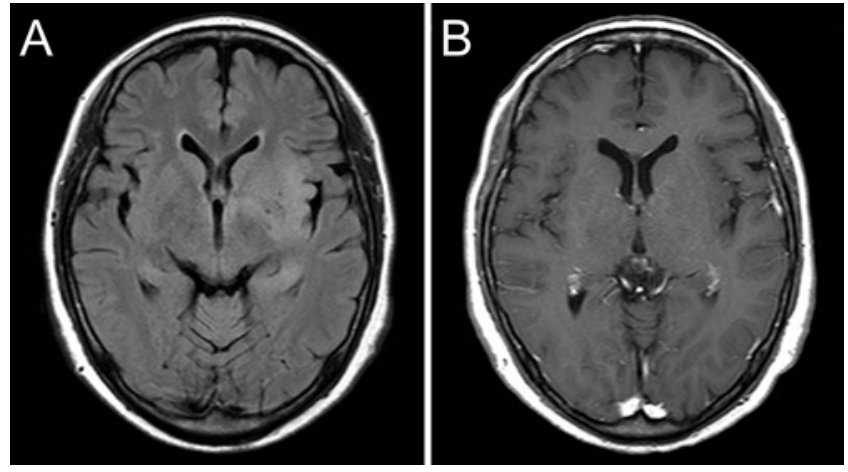

FIG. 1. First presentation. Axial FLAIR image (A) showing a high-intensity lesion in the left frontotemporal lobe. Axial gadolinium-enhanced T1-weighted image (B) demonstrating no enhanced lesion.
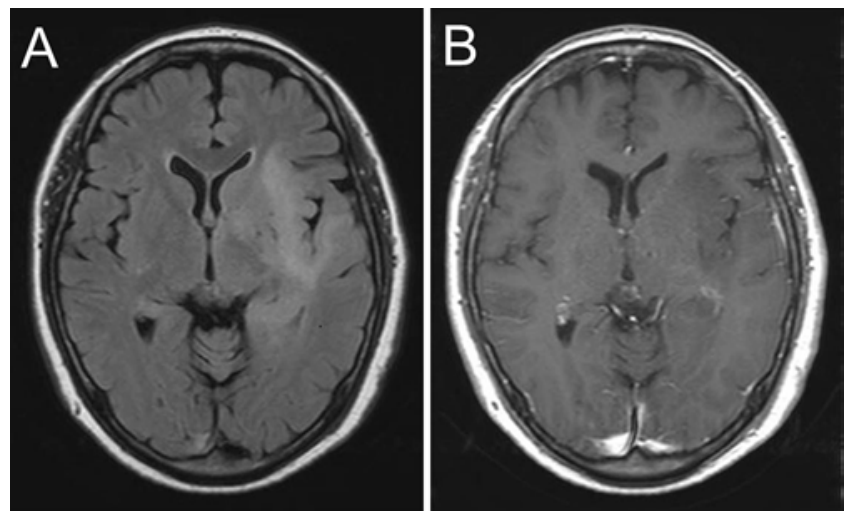

FIG. 2. Four years prior to current presentation. Axial FLAIR image showing progression in the high-intensity lesion (A). Axial gadoliniumenhanced T1-weighted image (B) showing no enhancement.

sentation had shown no progression, and the patient was followed up thereafter with annual MRI. Four years prior to the current presentation, we had noted progression in the high-intensity lesion on FLAIR imaging without enhancement on T1-weighted imaging (Fig. 2). An astrocytoma had been suspected, and our department had been consulted to consider biopsy for a definitive diagnosis. However, the patient had no complaints at that time, and no further invasive tests or treatments were conducted. No neurological impairment was observed until 3 months prior to the current presentation, and follow-up MRI had shown slow progression of the lesion. She then began to develop dysarthria and impaired short-term memory. These symptoms progressed, and she finally presented to our emergency department. Computed tomography revealed a novel hemorrhagic lesion in the right frontal lobe (Fig. 3A). Magnetic resonance imaging showed that the previously observed lesion in the left frontotemporal lobe and basal ganglia had further progressed (Fig. 3B), but no enhancement was observed on T1-weighted imaging (Fig. 3C). We started conservative treatment for the right frontal hemorrhage, and on day 30, we performed partial left temporal lobectomy to obtain a definitive diagnosis.

\section{Operation and Pathological Findings}

With the patient under general anesthesia, a left frontotemporal curvilinear skin incision was made and a small frontotemporal craniotomy was performed. After the dural incision, a corticotomy was performed within the inferior temporal gyrus, and partial lobectomy for the left temporal lesion was conducted toward the temporal base. After removal of the lesion, including the temporal tip, the dura mater was closed and the bone was fixed with titanium plates. Finally, the skin wound was closed. A specimen of the left temporal lobe was sent out for pathological examination (Fig. 4), and the diagnosis of diffuse astrocytoma was made.

\section{Postoperative Course}

The patient underwent postoperative rehabilitation. On day 65, follow-up MRI revealed novel gadoliniumenhanced lesions around the right carotid foramen (Fig. $5 \mathrm{~A}$ ), in the left parahippocampal gyrus (Fig. 5B), and in the right frontal lobe within the previous hemorrhagic lesion (Fig. 5C). Given the patient's history of lung cancer, we performed contrast-enhanced $\mathrm{CT}$ on day 70 because of suspected brain metastasis and primary lung lesion. A novel enhanced lesion in the right cervical lymph node (Fig. 6A) and massive bilateral pleural effusion (Fig. 6B) were noted with no apparent primary lesion in the lungs. First, we conservatively managed her progressive pleural effusion, but the left pleural effusion increased despite treat-
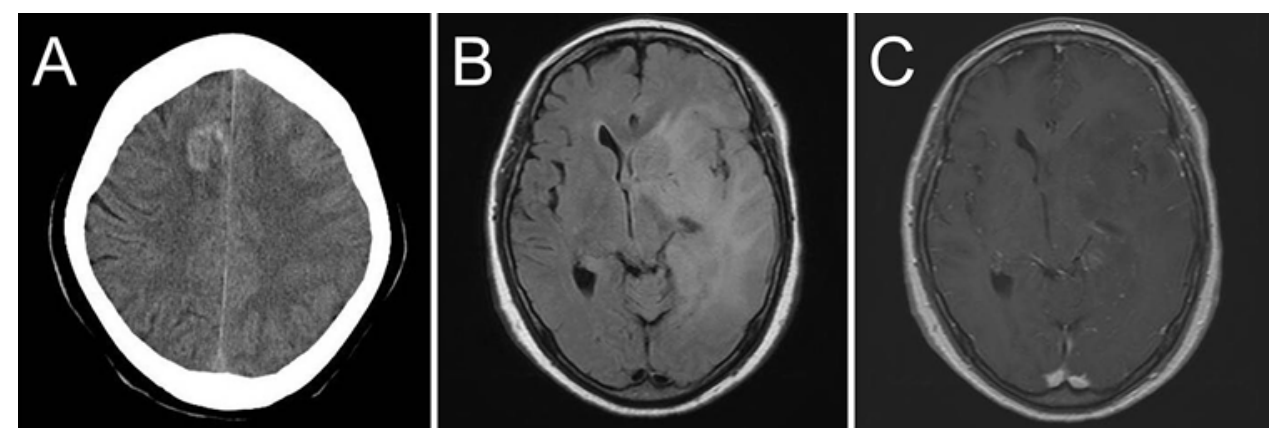

FIG. 3. Current presentation. Axial CT scan (A) demonstrating a new hemorrhagic lesion in the right frontal lobe. Axial FLAIR image (B) showing progression of the lesion in the left frontotemporal lobe. Axial gadolinium-enhanced T1-weighted image (C) showing no enhancement. 


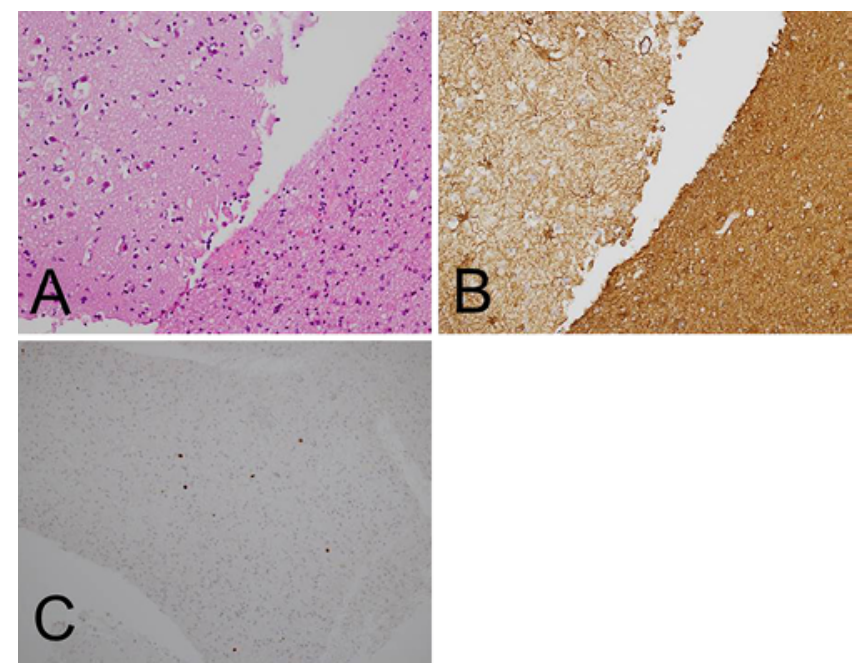

FIG. 4. Pathological findings of the specimen from the left temporal lobe showing mildly increased cellularity with mild atypia (A), positive GFAP (B), and low Ki-67 labeling index (C). H \& E (A).

ment. On day 85, we performed thoracocentesis for the left pleural effusion (Fig. 7A and B), and cytological examination of the pleural effusion revealed no atypical cells. On day 86 , a chest drainage tube was placed by our pulmonologist for repetitive accumulation of the left pleural effusion, and continuous drainage of the pleural effusion was started (Fig. 7C). The patient's impaired respiratory status continued to worsen with intractable accumulation of pleural fluid, and cytological examination of the massive pleural effusion was repeated on day 89 . The specimen exhibited malignant cells showing an increased nucleus/cytoplasm ratio, nuclear pleomorphism, and hyperchromatic nuclei (Fig. 8), and we suspected a small round cell tumor such as lymphoma. On day 93, we collected another specimen of the massive pleural effusion and examined it using the cell block technique. On day 94, the patient's respiratory status further worsened despite continuous pleural fluid drainage, and she died. After her death, glioblastoma was diagnosed using the cell block specimen of pleural effusion (Fig. 9), which showed the following characteristics: glial fibrillary acidic protein (GFAP; +), epithelial membrane antigen (-), AE1/AE3 (-), leukocyte common antigen (LCA; -), S100 $(-)$, CD56 (+), synaptophysin (-), and chromogranin A (-).
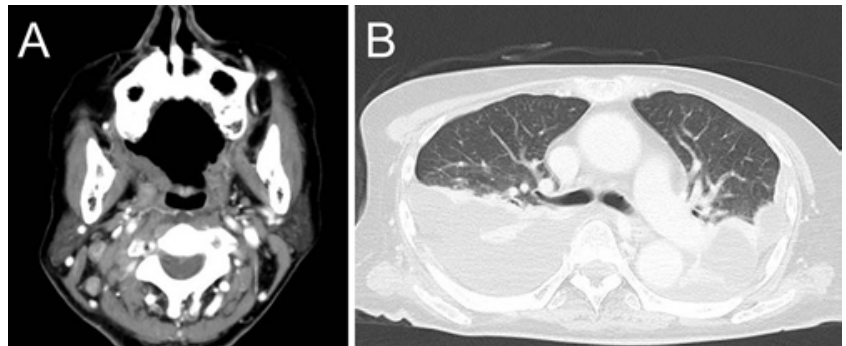

FIG. 6. Axial contrast-enhanced CT shows a novel lesion in the right cervical lymph node $(\mathbf{A})$ and bilateral pleural effusion (B).

\section{Discussion \\ Extracranial Glioblastoma and Its Increased Incidence}

Several studies have revealed the involvement of multiple organs, including the lung and pleura, in patients with metastatic glioblastoma. ${ }^{2,6,15-19}$ Patients in these studies were mainly diagnosed by histological examination of biopsy specimens. The incidence of extracranial glioblastoma is considered to be increasing. ${ }^{8}, 12,18$ The mean survival time of patients with glioblastoma is very short, while systemic metastasis seems to take a considerable period of time to emerge. Recent treatments such as temozolomide can contribute to prolonging survival time, ${ }^{7,14}$ but such therapy may also contribute to the prolonged development of circulating malignant cells and thus the increased incidence of extracranial metastasis of glioblastoma. Therefore, early diagnosis and treatment would improve the clinical course of such patients.

\section{Diagnosis of Metastatic Lung and Pleural Glioblastoma}

A previous study showed that the lung and pleura are two of the most frequent sites of systemic metastasis of glioblastoma. ${ }^{8,12}$ Chivukula et al. ${ }^{2}$ described a patient with extracranial glioblastoma diagnosed by fine needle aspiration biopsy. In that report, the patient had a mass in the upper lobe of the right lung, and malignant cells were found on examination of a specimen from that mass but not on examination of the bronchoalveolar lavage fluid or pleural fluid. In the present case, the first cytological analysis using pleural effusion obtained by thoracocentesis was also negative, but repeated examination revealed the presence of glioblastoma cells in the pleural effusion.
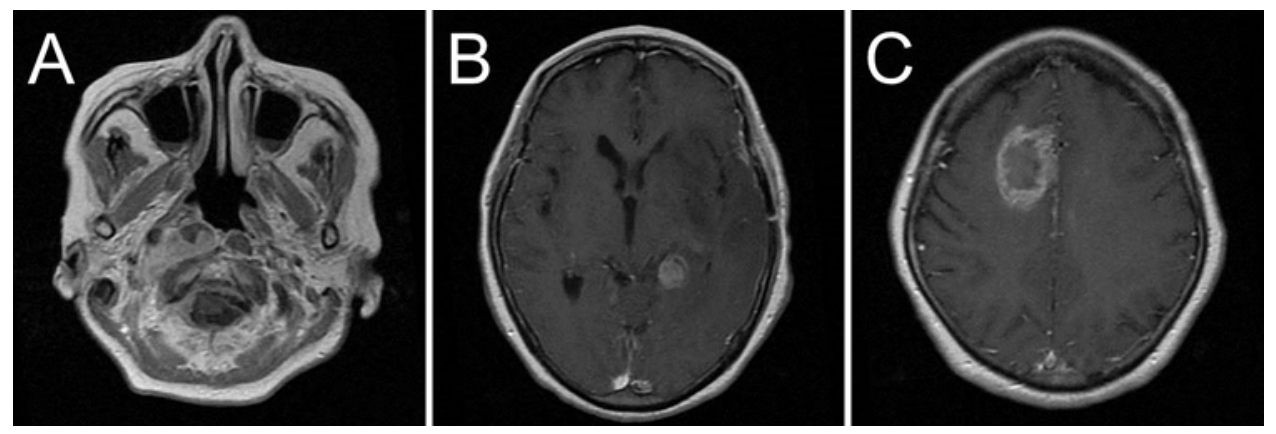

FIG. 5. Axial gadolinium-enhanced T1-weighted images obtained on day 65 , showing novel lesions around the right carotid foramen (A), in the left parahippocampal gyrus (B), and in the right frontal lobe within the previous hemorrhagic lesion (C). 


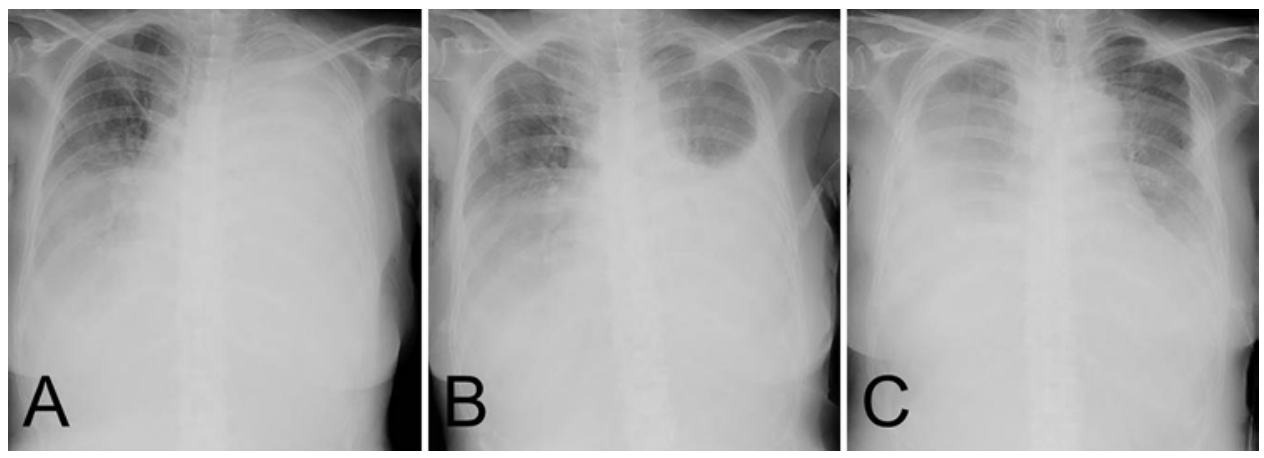

FIG. 7. Chest radiographs obtained before $(\mathbf{A})$ and after $(\mathbf{B})$ thoracocentesis and after placement of the chest drainage tube (C).

Thus, in patients with glioma, progressive accumulation of pleural fluid warrants repeated cytological examination to increase its sensitivity.

\section{Difficulty of Diagnosing Malignant Disease by Cytological Analysis}

Several reports have documented the difficulty of diagnosing glioblastoma cells by cytological analysis ${ }^{2,11}$ and the possibility of misinterpreting glioblastoma cells as poorly differentiated non-small cell lung carcinoma cells because both are often small with hyperchromatic nuclei, an increased nucleus/cytoplasm ratio, and prominent pleomorphism. ${ }^{1,5}$ These reports have also demonstrated the significance of immunostaining these cells, which show the following immunostaining profile: GFAP $(+)$, cytokeratin

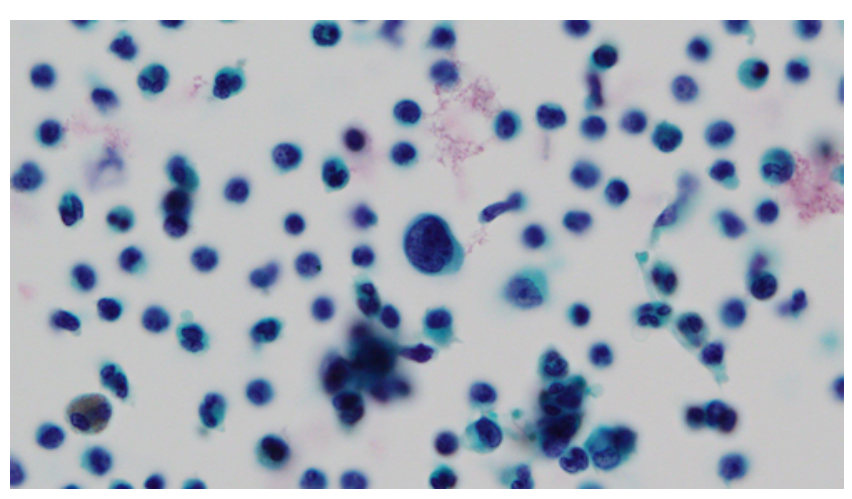

FIG. 8. Papanicolaou staining of the left pleural effusion sample showing increased nucleus/cytoplasm ratio, nuclear pleomorphism, and hyperchromatic nuclei.

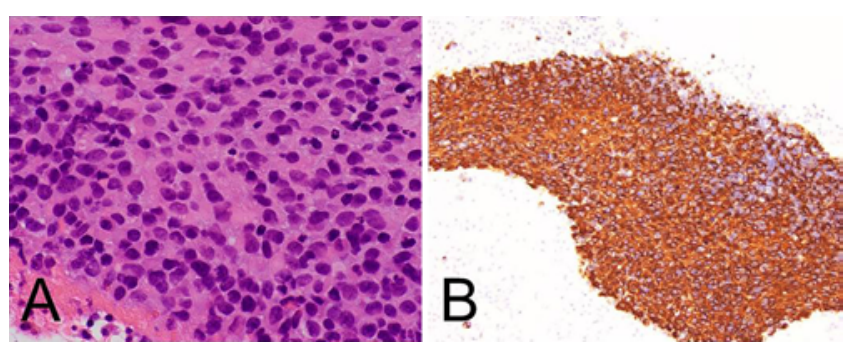

FIG. 9. Cytological analysis of the left pleural effusion sample using the cell block technique. $H \& E(A)$ and GFAP (B).
$(-)$, chromogranin (-), and LCA (-). In the present case, cytological analysis of the pleural fluid cells suggested a small round cell tumor, making the diagnosis difficult. However, our cytological examination also showed the following immunostaining profile: GFAP $(+)$, cytokeratin $(-)$, chromogranin (-), and LCA (-). These findings made the diagnosis of glioblastoma reasonable.

\section{Usefulness of the Cell Block Technique for Low-Density Specimens}

The cell block technique reportedly increases the sensitivity of cytological analysis by decreasing cellular dispersion when using fluid specimens such as pleural and peritoneal effusions..$^{10,13,21}$ Wardeh et al. ${ }^{20}$ also described the usefulness of this technique when no abnormality had been detected by imaging studies such as CT. Indeed, our patient had no apparent intrathoracic lesion on CT, but the technique still revealed the presence of glioblastoma cells in a pleural sample. Our patient's impaired respiratory status rapidly progressed in a very short time. Earlier diagnosis might have contributed to initiation of earlier treatment and improvement in the clinical course.

\section{Conclusions}

In patients with a history of glioma, the presence of extremely intractable pleural effusion may suggest pleural or lung metastasis. In such patients, immediate diagnosis by cytological analysis of pleural effusion using the cell block technique should be considered to initiate appropriate chemotherapy regardless of the positive or negative results of imaging studies.

\section{References}

1. al-Rikabi AC, al-Sohaibani MO, Jamjoom A, al-Rayess MM: Metastatic deposits of a high-grade malignant glioma in cervical lymph nodes diagnosed by fine needle aspiration (FNA) cytology - case report and literature review. Cytopathology 8:421-427, 1997

2. Chivukula M, Dincer HE, Biller JA, Krouwer HG, Simon G, Shidham V: FNAB cytology of extra-cranial metastasis of glioblastoma multiforme may resemble a lung primary: a diagnostic pitfall. Cytojournal 2:9, 2005

3. Cho JS, Kim GE, Lee JS, Lee JH, Nam JH, Choi C: Diagnostic usefulness of MUC1 and MUC4 for distinguishing between metastatic adenocarcinoma cells and reactive meso- 
thelial cells in effusion cell blocks. Acta Cytol 57:377-383, 2013

4. García Carretero R, Manotas-Hidalgo M, Romero Brugera M, El Bouayadi Mohamed L: Pleural effusion of malignant aetiology: cell block technique to establish the diagnosis. BMJ Case Rep 2016:bcr2016215140, 2016

5. González Cámpora R, Otal Salaverri C, Vázquez Ramirez F, Salguero Villadiego M, Galera Davidson H: Metastatic glioblastoma multiforme in cervical lymph nodes. Report of a case with diagnosis by fine needle aspiration. Acta Cytol 37:938-942, 1993

6. Greif J, Horovitz M, Marmor S: Pleuropulmonary metastasis from an intracranial glioblastoma. Lung Cancer 20:135137, 1998

7. Hegi ME, Diserens AC, Gorlia T, Hamou MF, de Tribolet N, Weller M, et al: MGMT gene silencing and benefit from temozolomide in glioblastoma. N Engl J Med 352:997-1003, 2005

8. Kalokhe G, Grimm SA, Chandler JP, Helenowski I, Rademaker A, Raizer JJ: Metastatic glioblastoma: case presentations and a review of the literature. J Neurooncol 107:21-27, 2012

9. Minagawa T, Murata Y, Uchikawa S, Uehara T: Malignant pericardial tamponade in a patient with hormone-refractory prostate cancer. Int J Clin Oncol 15:101-103, 2010

10. Nakayama Y, Nakagomi H, Omori M, Inoue M, Takahashi $\mathrm{K}$, Maruyama M, et al: Benefits of using the cell block method to determine the discordance of the HR/HER2 expression in patients with metastatic breast cancer. Breast Cancer 23:633-639, 2016

11. Nauen DW, Li QK: Cytological diagnosis of metastatic glioblastoma in the pleural effusion of a lung transplant patient. Diagn Cytopathol 42:619-623, 2014

12. Piccirilli M, Brunetto GM, Rocchi G, Giangaspero F, Salvati M: Extra central nervous system metastases from cerebral glioblastoma multiforme in elderly patients. Clinico-pathological remarks on our series of seven cases and critical review of the literature. Tumori 94:40-51, 2008

13. Rivero ER, Grando LJ, Menegat F, Claus JD, Xavier FM: Cell block technique as a complementary method in the clinical diagnosis of cyst-like lesions of the jaw. J Appl Oral Sci 19:269-273, 2011

14. Stupp R, Mason WP, van den Bent MJ, Weller M, Fisher B, Taphoorn MJ, et al: Radiotherapy plus concomitant and adjuvant temozolomide for glioblastoma. N Engl J Med 352:987-996, 2005
15. Taskapilioglu MO, Aktas U, Eser P, Tolunay S, Bekar A: Multiple extracranial metastases from secondary glioblastoma: a case report and review of the literature. Turk Neurosurg 23:824-827, 2013

16. Yasuhara T, Tamiya T, Meguro T, Ichikawa T, Sato Y, Date I, et al: Glioblastoma with metastasis to the spleen-case report. Neurol Med Chir (Tokyo) 43:452-456, 2003

17. Yokoyama H, Ono H, Mori K, Kishikawa M, Kihara M: Extracranial metastasis of glioblastoma with sarcomatous component. Surg Neurol 24:641-645, 1985

18. Waite KJ, Wharton SB, Old SE, Burnet NG: Systemic metastases of glioblastoma multiforme. Clin Oncol (R Coll Radiol) 11:205-207, 1999

19. Wakamatsu T, Matsuo T, Kawano S, Teramoto S, Matsumura $\mathrm{H}$ : Glioblastoma with extracranial metastasis through ventriculopleural shunt. Case report. J Neurosurg 34:697-701, 1971

20. Wardeh R, Lee JG, Gu M: Endoscopic ultrasound-guided paracentesis of ascitic fluid: a morphologic study with ultrasonographic correlation. Cancer Cytopathol 119:27-36, 2011

21. Zhou J, Yao H, Zhao J, Zhang S, You Q, Sun K, et al: Cell block samples from malignant pleural effusion might be valid alternative samples for anaplastic lymphoma kinase detection in patients with advanced non-small-cell lung cancer. Histopathology 66:949-954, 2015

\section{Disclosures}

The authors report no conflict of interest concerning the materials or methods used in this study or the findings specified in this paper.

\section{Author Contributions}

Conception and design: Hori. Acquisition of data: Hori, Fukuhara, Aoi. Analysis and interpretation of data: Hori, Fukuhara, Aoi, Shinno. Drafting the article: Hori. Critically revising the article: all authors. Reviewed submitted version of manuscript: all authors. Approved the final version of the manuscript on behalf of all authors: Hori. Administrative/technical/material support: Fukuhara, Aoi, Oda. Study supervision: Fukuhara, Aoi.

\section{Correspondence}

Yusuke S. Hori: National Hospital Organization Okayama Medical Center, Okayama, Japan. ys-hori@umin.ac.jp. 